\title{
Proposal of MIMO Ultra-Wide Band Antenna with Low Mutual Coupling
}

\author{
Le Trong Trung, Nguyen Quoc Dinh, Ta Chi Hieu \\ Faculty of Radio-Electronics, Le Quy Don Technical University, Vietnam \\ Correspondence: Nguyen Quoc Dinh, dinhnq@mta.edu.vn \\ Manuscript communication: received 28 July 2014, accepted 15 April 2015
}

\begin{abstract}
In this paper, a new ultra-wide band (UWB) MIMO antenna is proposed. A MIMO antenna set consists of two single ultra-wide band antennas. This simple and compact MIMO antenna, which is designed to work from $3.1 \mathrm{GHz}$ to $10.6 \mathrm{GHz}$, has a broad bandwidth with the VSWR $\leq 2$. In addition, MIMO antenna characteristics such as radiation pattern, maximal gain are thoroughly investigated.
\end{abstract}

Keywords- Ultra-Wide Band (UWB), Ultra-Wide Band antenna, printed antenna, planar antenna, MIMO antenna.

\section{INTRODUCTION}

MIMO (Multi-Input Multi-Output) systems use multiple antennas at both transmitter and receiver sides. As a result, these systems can obtain transmit and receive diversity to improve the system performance or perform transmit as well as receive beamforming to enhance the system efficiency and suppress interferences. Moreover, the system capacity can be significantly improved thank to spatial multiplexing. In MIMO systems, equal numbers of transmit and receive antennas, such as $2 \times 2$, $4 \times 4$, are often chosen.

The ultra-wide band (UWB) technology was proposed to achieve high data rates in short range communications. To further enhance the data rates and cover ranges of the UWB communication systems, the combination of MIMO techniques and the UWB technology has gain considerable attention recently. A MIMO UWB antenna, however, often uses many radiation elements which have considerable mutual coupling between each other. This phenomenon is considered as a bottleneck in the MIMO UWB systems and should be minimized to improve the stability and radiation efficiency of the whole system. Thus, the designing of a MIMO UWB antenna is not only the designing of its elements but the mutual coupling must also be taken into account.

There have been a number of UWB antennas with different structures and materials including defected ground structure plane radiator [1], UWB monopole antenna with semi-ellipse shaped patches [2], slot UWB antenna with half-wavelength and quarter-wavelength slot on the radiation plane [3], defected ground structure [4], dipole antennas [5,6], printed antennas [7], printed antennas with defected ground structure [8], slot antennas [9]. In [10], the authors have also proposed an ultra-wide antenna with slotted rectangular radiation plane to achieve small-sized structure.

Some designs of the MIMO UWB antennas with two elements using different structures and materials have also been proposed. In [11], Najam et al. proposed a MIMO antenna composed of two rounded antennas with radius $R=12 \mathrm{~mm}$, antenna dimensions are of $80 \times 34 \times 0.8 \mathrm{~mm}^{3}$ on FR4 dielectric substrate. Compared to this structure, the design in [12] by Li et al. has smaller size but more complex structure.

In this paper, a MIMO UWB antenna with small, compact and thin structure, suitable for general structure of UWB equipment, is proposed. The structure of the antenna elements is fabricated on the FR4 substrate with dielectric permittivity of $\varepsilon_{r}=4.4$ and loss factor of $\tan \delta=0.02$. Since the mutual coupling between elements in MIMO antenna always exists, it is necessary to calculate and tune the parameters of the antenna elements, set suitable distance between them and select suitable size of the dielectric substrate so that the proposed MIMO UWB antenna can achieve low mutual coupling while keeping the desired voltage standing wave ratio (VSWR $\leq 2)$, isotropic radiation pattern and good gain. To minimize the mutual coupling between antennas, a stub is placed between two elements in the MIMO antenna. Finally, the prototype was fabricated and its characteristics were measured and compared with simulation results.

\section{Design of MIMO UWB Antenna}

\subsection{Structure of Proposed Antenna}

In the first step of designing the MIMO UWB antenna, its single UWB element is calculated. Considering the design in [13] by Kumar et al.: a substrate of FR4 with $\varepsilon_{r}=4.3$ and $\tan \delta=0.02$ was used; the dimensions are $60 \times 45 \times 1.6 \mathrm{~mm}^{3}$ and ground plane made of copper with the thickness of $35 \mu \mathrm{m}$, however, that antenna structure is fairly big. Based on the structure in [13], the authors proposed an antenna 
with smaller size, more compact and simpler structure that serves as radiation elements for the MIMO UWB antennas.

Based on the working frequency range of the antenna, particularly the wavelength at resonance frequency $f_{0}$ and the electric permittivity $\varepsilon_{r}$, the authors calculated preliminary dimensions of antenna radiation patch.

The width of radiation patch is

$$
w_{3}=\frac{c}{2 f_{0}} \sqrt{\frac{2}{\varepsilon_{\rho}+1}} .
$$

The length of radiation patch is

$$
l_{1}=l_{\text {eff }}-2 \Delta l
$$

where

$$
\begin{gathered}
\Delta l=0,412 h \frac{\left(\varepsilon_{\text {eff }}+0,3\right)\left(\frac{w_{3}}{h}+0,264\right)}{\left(\varepsilon_{\text {eff }}-0,258\right)\left(\frac{w_{3}}{h}+0,8\right)}, \\
l_{\text {eff }}=\frac{c}{2 f_{0} \sqrt{\varepsilon_{\text {eff }}}}, \\
\varepsilon_{\text {eff }}=\frac{\varepsilon_{r}+1}{2}+\frac{\varepsilon_{r}-1}{2}\left[1+\frac{10 h}{w_{3}}\right]^{-\frac{1}{2}} .
\end{gathered}
$$

With relative permittivity of $\varepsilon_{r}=4.4$, frequency of $f_{0}=7.0 \mathrm{GHz}$ and the thickness of dielectric substrate $h=1.6 \mathrm{~mm}$, the length and the width of radiation patch are $l_{1}=11.5 \mathrm{~mm}$ and $w_{3}=13.4 \mathrm{~mm}$, respectively. These are preliminary dimensions of a narrow frequency band antenna. With broadband antennas, if the width of radiation patch is bigger, frequency range will be widened because of increase in radiation emission and in amplification ratio as a result of increased emission area. Therefore, with UWB antennas, the width of radiation patch should be increased to achieve expected frequency band. When the width of radiation plane is enlarged, its length must also be increased accordingly. In addition, the square hole is pierced and dimensions of the antenna are adjusted to create multi-resonance, which helps to expand the frequency range and reduce the dimensions of the antenna. Finally, the suitable dimensions of the radiation plane are selected as $l_{1}=14$ $\mathrm{mm}$ and $w_{3}=15 \mathrm{~mm}$.

For the purpose of calculating the width of the antenna feeding strip $\left(w_{1}\right)$, the following formula in [14, 15] is used:

$$
Z_{0}=\frac{87}{\sqrt{\varepsilon_{r}+1,41}} \ln \left(\frac{5,98 h}{0,8 w_{1}+t}\right),
$$

where $Z_{0}$ is the characteristic impedance of the line, set to $Z_{0}=50 \Omega ; \varepsilon_{r}$ is the relative permittivity; $h$ is the thickness of dielectric substrate; $t$ is the thickness of copper layer.

Based on the formula for $Z_{0}$, the width of feeding strip is calculated as $w_{1} \approx 3 \mathrm{~mm}$. From the design of the single element, by using a slow adjustment method to adjust parameters of antenna, the authors have selected suitable MIMO UWB antenna with geometrical parameters given in Table I.
Table I

The Optimal Geometrical Parameters (MM)

\begin{tabular}{|c|c|c|c|c|c|}
\hline Parameters & Values & Parameters & Values & Parameters & Values \\
\hline$L$ & 30 & $l_{1}$ & 14 & $w_{3}$ & 15 \\
\hline$W$ & 70 & $l_{2}$ & 2 & $w_{4}$ & 2 \\
\hline$W_{g}$ & 32 & $l_{3}$ & 1 & $w_{5}$ & 2 \\
\hline$L_{g}$ & 10 & $l_{4}$ & 11 & $w_{6}$ & 2 \\
\hline$s$ & 5 & $l_{5}$ & 1 & $w_{7}$ & 0.5 \\
\hline$t$ & 0.035 & $l_{6}$ & 4 & $w_{8}$ & 1 \\
\hline$h$ & 1.6 & $l_{7}$ & 10 & $w_{9}$ & 1.5 \\
\hline$d_{1}$ & 23 & $w_{1}$ & 3 & $w_{10}$ & 3 \\
\hline$d_{2}$ & 6 & $w_{2}$ & 8.5 & $d$ & 38 \\
\hline
\end{tabular}

\subsection{Simulation Results}

The simulations were performed under the condition that both elements in MIMO antenna are working concurrently. With no stub, the simulated VSWR is shown in Figure 2. In order to reduce the mutual coupling effect between antennas, a copper stub on the plane of the MIMO antenna is added to separate its two elements. The VSWR of the MIMO UWB antenna with attached stub is shown in Figure 3. As can be seen from the figure, in case of using the stub the VSWR is smaller than that of the case of no stub and the VSWR is less than 2 in the whole frequency range for both antenna elements. In the case of no stub between two elements, when one antenna works, it will induce the other, causing a considerable mutual coupling effect between the two elements because the gap between them is not big enough. If a stub is used then when one antenna works, it will induce the stub first, therefore the induction to the other antenna will be reduced. As a result, the mutual coupling effect between two antennas decreases. The induced current on the stub will create a secondary electro-magnetic field which in turn will have an effect on the two antenna elements, this will affect not only the mutual coupling between the two antennas but also their VSWR. Therefore, the dimensions of the stub must be carefully selected. In the proposed MIMO antenna, the authors have selected the stub as shown in Figure 1.

The mutual coupling effect between two antennas is given in the following formula [16]:

$$
\begin{aligned}
\rho_{e} & =\left|r_{12}\right| \\
& =\frac{\left|S_{11}^{*} \cdot S_{12}+S_{21}^{*} \cdot S_{22}\right|^{2}}{\left(\sqrt{1-\left|S_{11}\right|^{2}-\left|S_{21}\right|^{2}}\right)\left(\sqrt{1-\left|S_{22}\right|^{2}-\left|S_{12}\right|^{2}}\right)} .
\end{aligned}
$$

In order to ensure that MIMO antennas work properly when the voltage standing wave ratio is less than 2 (equivalent to return loss ratio $S_{11}$ and $S_{22}<10 \mathrm{~dB}$ ) it is required that $\rho_{e}<0.5$ [17]; this is equivalent to the fact that $S_{12}$ and $S_{21}$ are less than $-15 \mathrm{~dB}$.

Figure 4 shows the simulated values of $S_{12}$ and $S_{21}$ of MIMO antenna in two cases: with and without the stub. To keep the mutual coupling effect between elements low, it is required that $S_{12}$ and $S_{21}$ are less than $-15 \mathrm{~dB}$. From Figure 4 it is apparent that in the case of using the stub, $S_{12}$ and $S_{21}$ are less than $-15 \mathrm{~dB}$ in the whole investigated frequency range while without the stub, $S_{12}$ and $S_{21}$ are greater than $-15 \mathrm{~dB}$ in the frequency range from $3 \mathrm{GHz}$ to $5 \mathrm{GHz}$. 


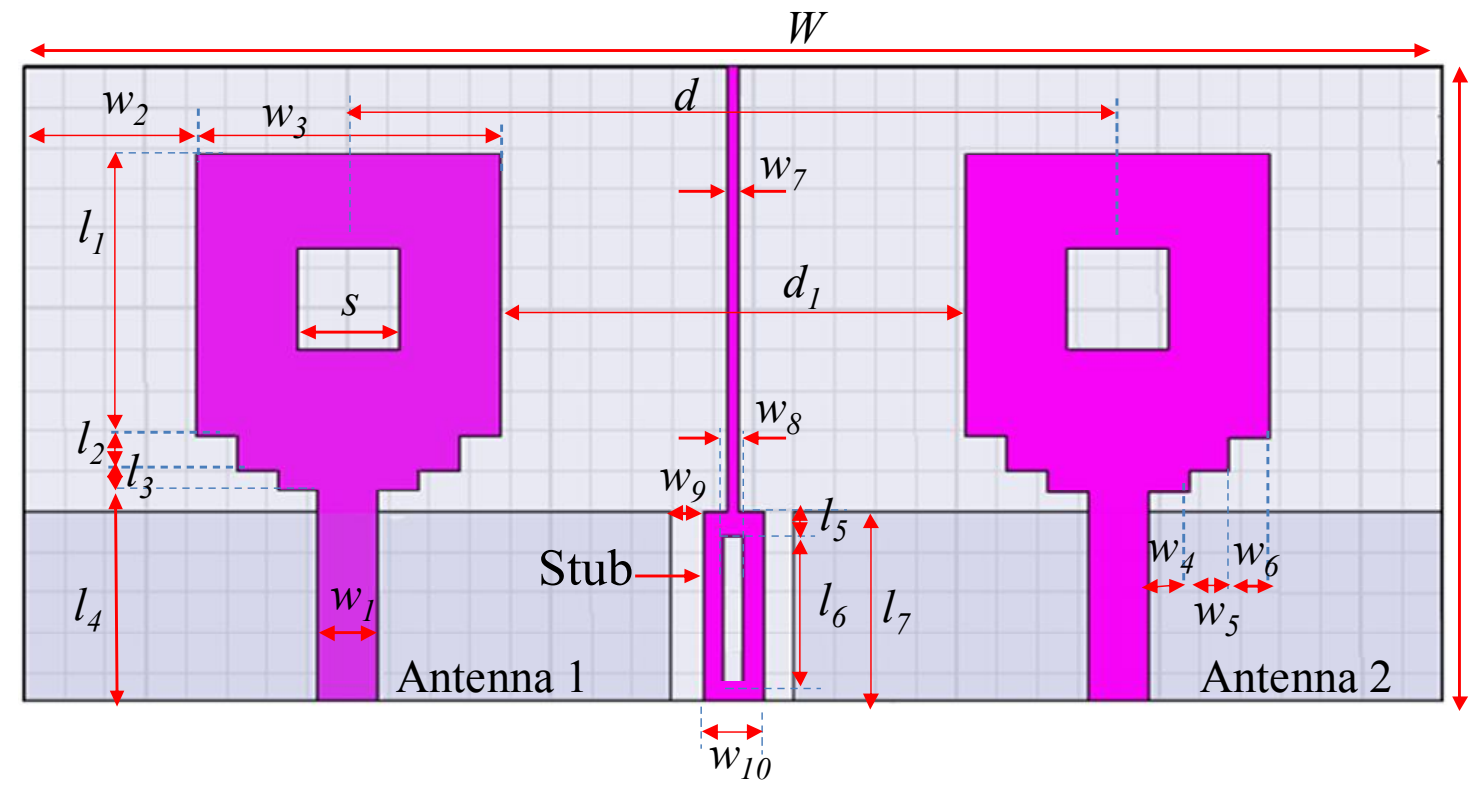

(a)

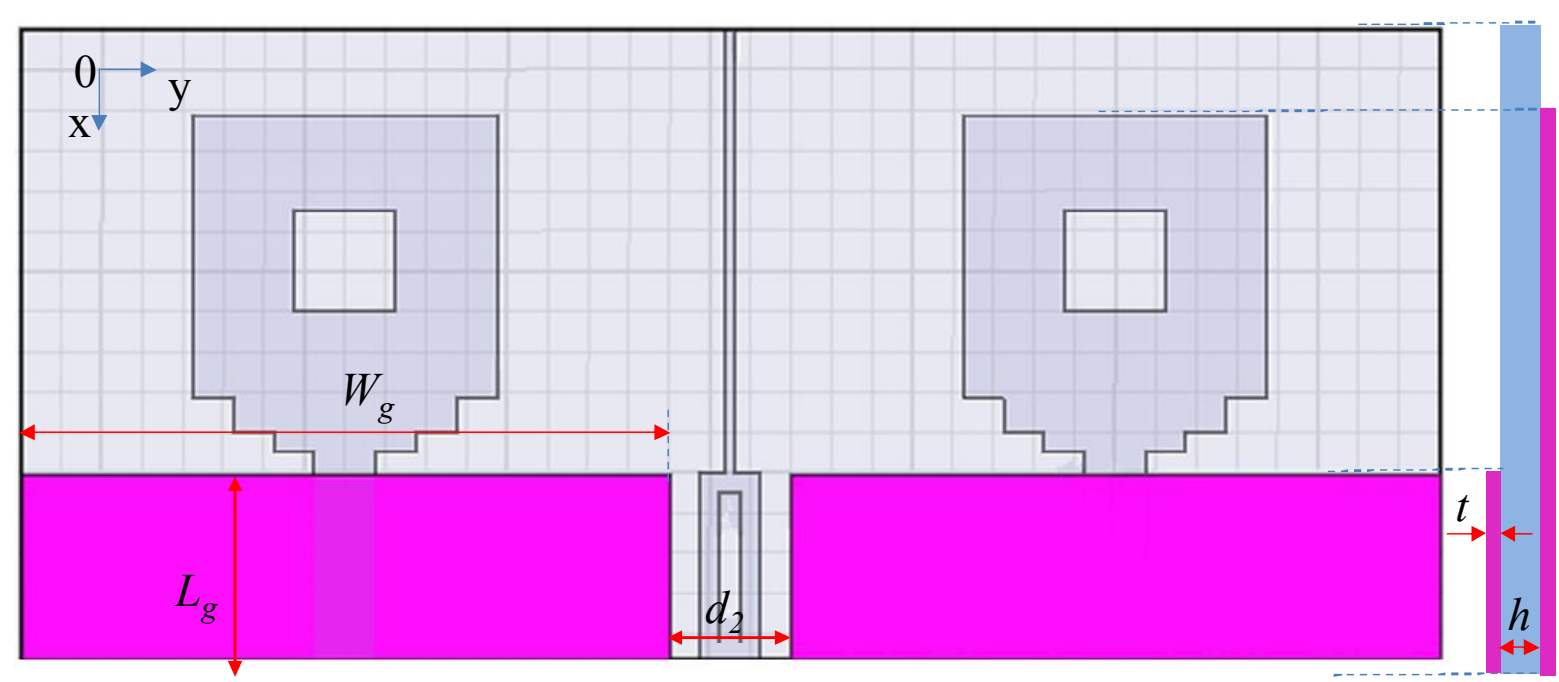

(b)

Figure 1. Configuration of MIMO antenna: (a) top view; (b) bottom view; (c) side view.

In the case of using the stub, after completing the simulation and calculation based on formula (1), we got the graph showing the values of $\rho_{e}$ in working frequency range in Figure 5. From Figure 5, it is clear that the mutual coupling ratio between two antennas is small, this satisfies the requirement for the two elements of the proposed MIMO UWB antenna to work properly.

To better understand the mutual coupling effect between the two elements in the MIMO antenna, the surface current distribution is investigated in the case when antenna 1 works while antenna 2 does not. The current distribution is shown in Figure 6, in which one can see if the stub structure is not used, the current of antenna 1 induces that of antenna 2. However, when the stub structure is used between 2 antennas, the induced current between the two antennas decreases significantly. Instead, the induced current will be distributed mainly on the stub.

Thus, when the two antennas are placed at distance of $d=38 \mathrm{~mm}=0.38 \lambda_{\max }$ (with $\lambda_{\max }=c / f_{\min }$ ) from each other, the use of the additional stub helps these antennas work properly with little mutual coupling effect.

It is clear that the use of the stub can reduce mutual coupling effect between the two elements in the proposed MIMO antenna, we therefore will use this structure and investigate other parameters of the MIMO antenna.

Since the frequency range is very wide, the radiation 


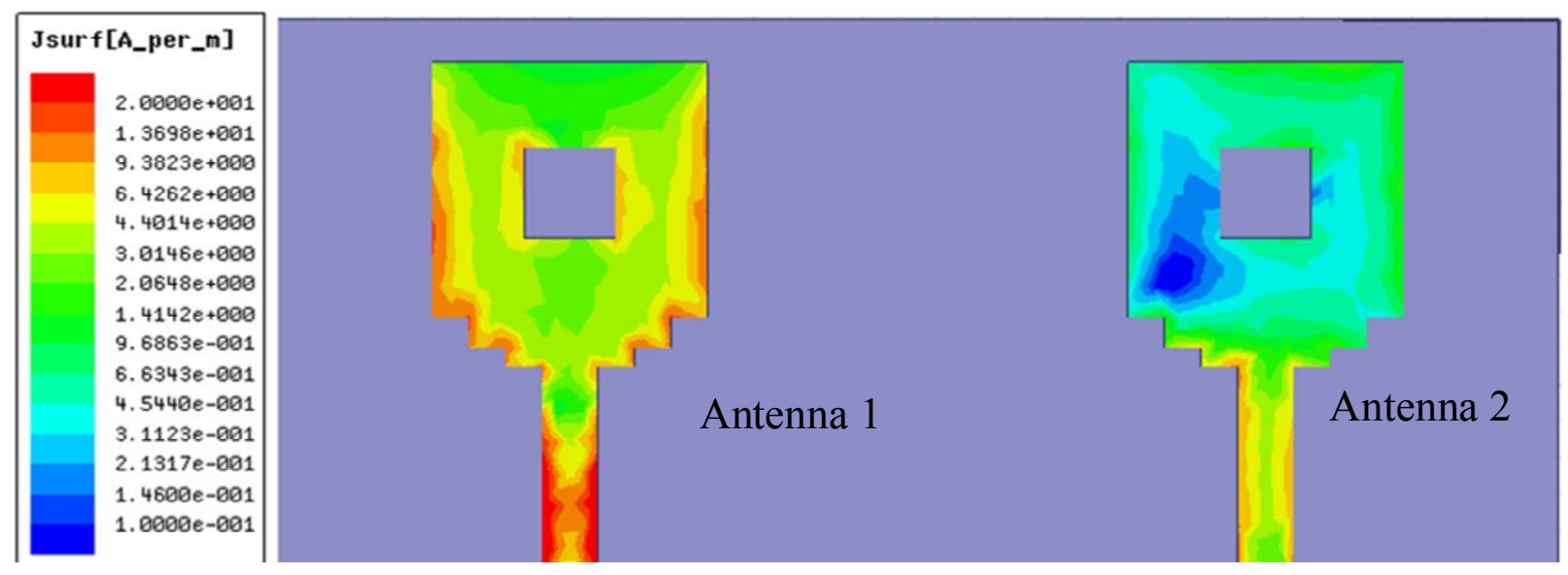

(a)

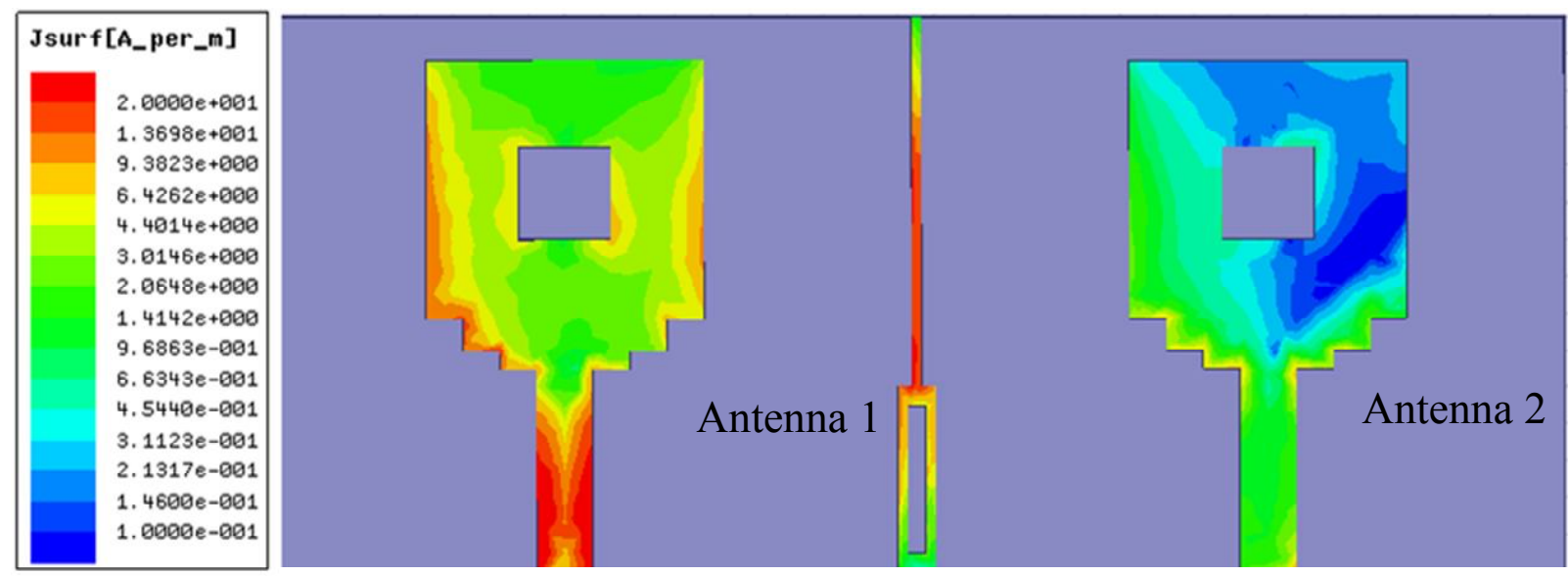

(b)

Figure 6. Current allocation at frequency $4.0 \mathrm{GHz}$ (a) without the stub; (b) with the stub.

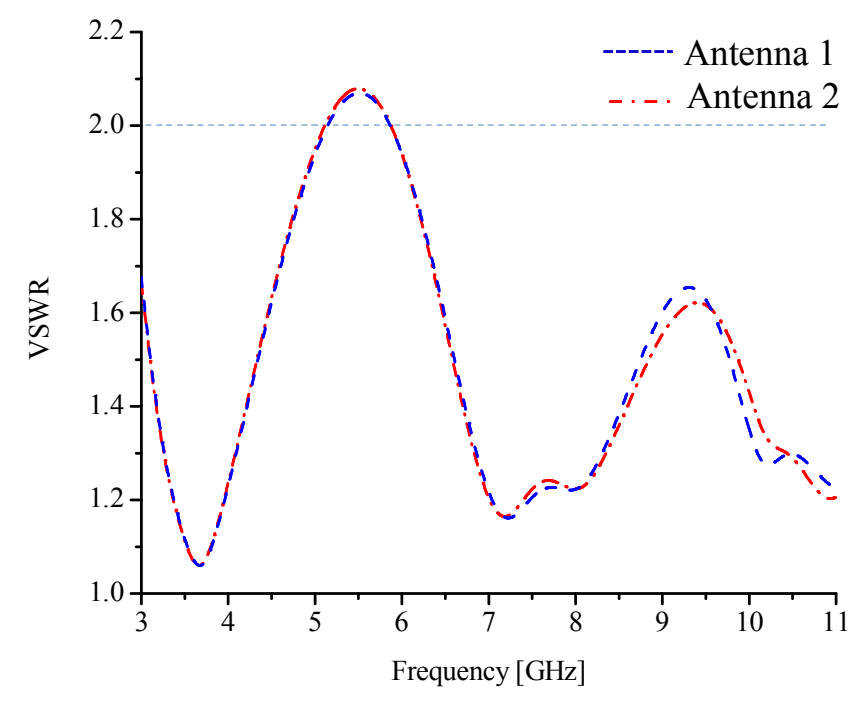

Figure 2. VSWR of MIMO elements without the stub.

pattern of the antenna needs to be simulated at different frequencies. The radiation pattern of MIMO antenna with both elements working is shown in Figure 7. The patterns at the frequencies of $3.1 \mathrm{GHz}, 5.0 \mathrm{GHz}, 7.0$ $\mathrm{GHz}, 9.0 \mathrm{GHz}$ and $10.6 \mathrm{GHz}$ are shown on Figures

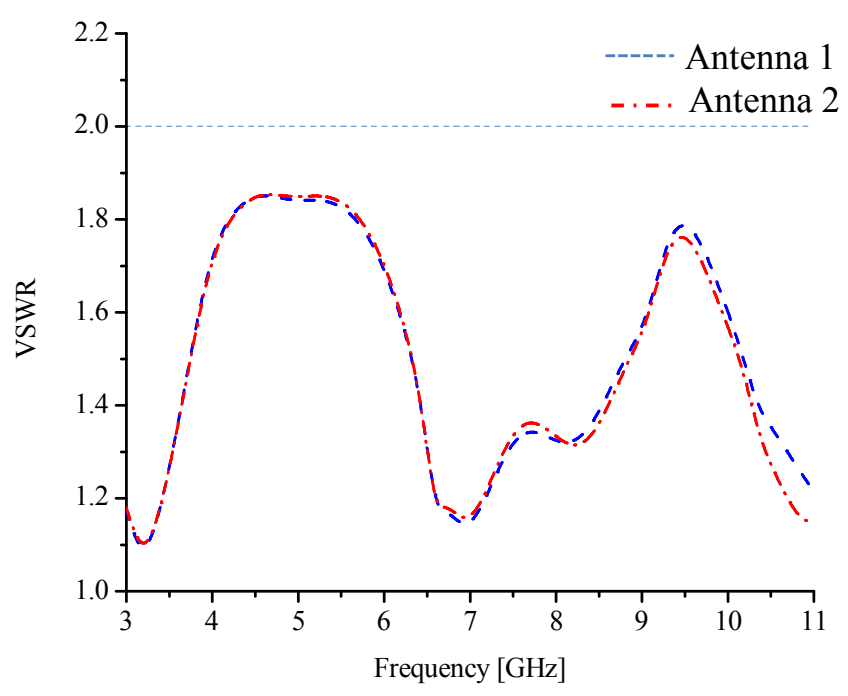

Figure 3. VSWR of MIMO elements with the stub.

7(a), (b), (c), (d), (e), respectively. In particular, solid line shows the pattern in the xoz plane, the dashed line shows the pattern in the yoz plane. From Figure 7, it can be seen that the radiation pattern of the MIMO antenna is nearly isotropic and varies within the frequency 


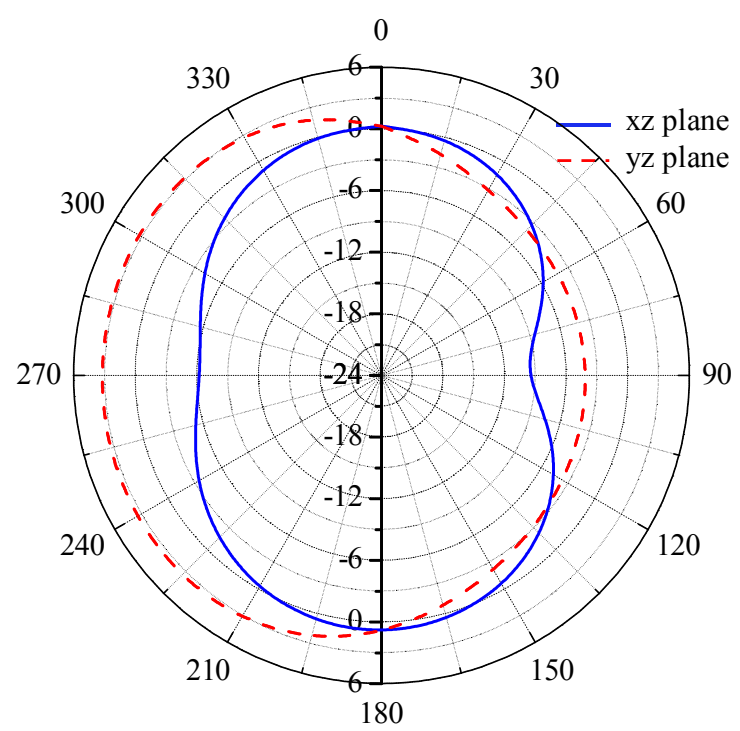

(a)

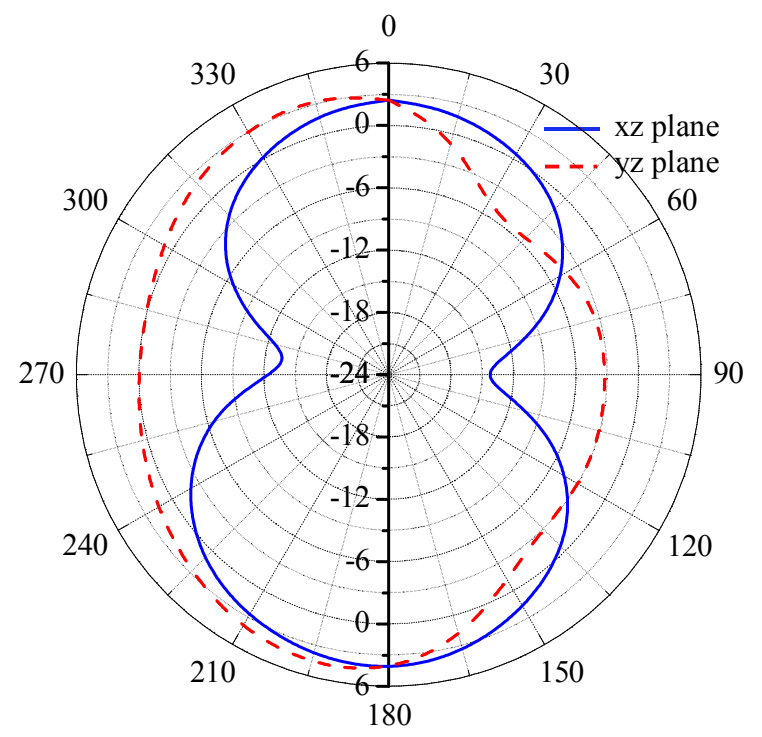

(b)

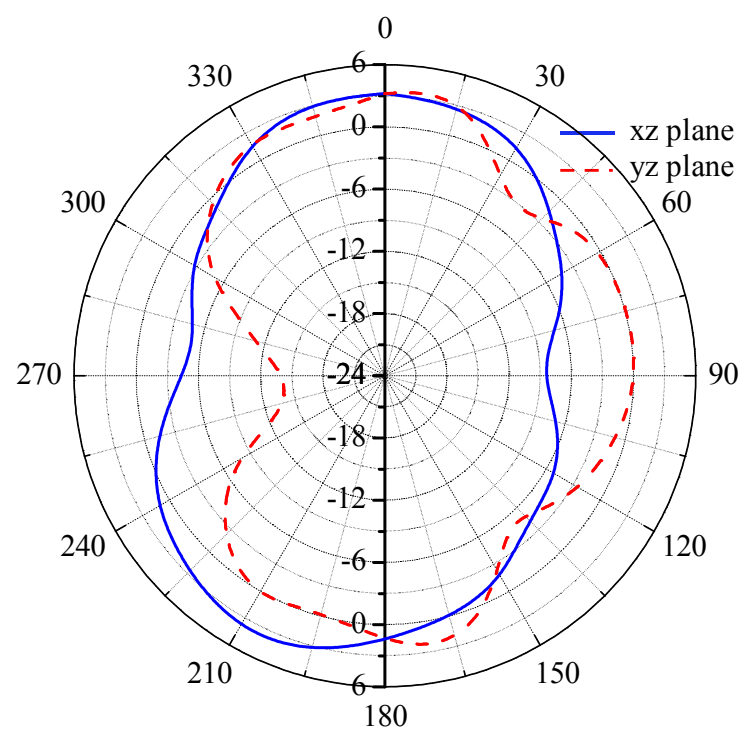

(c)

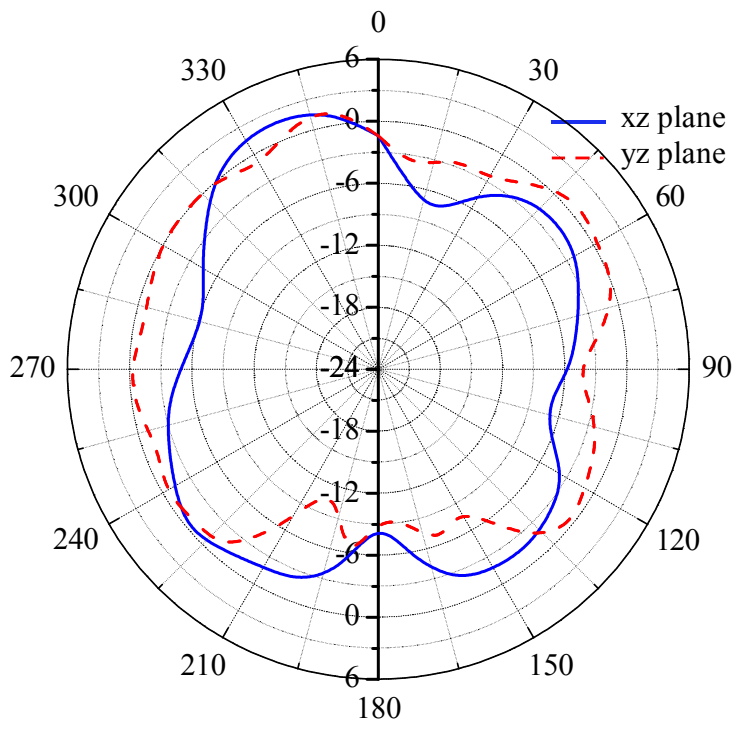

(d)

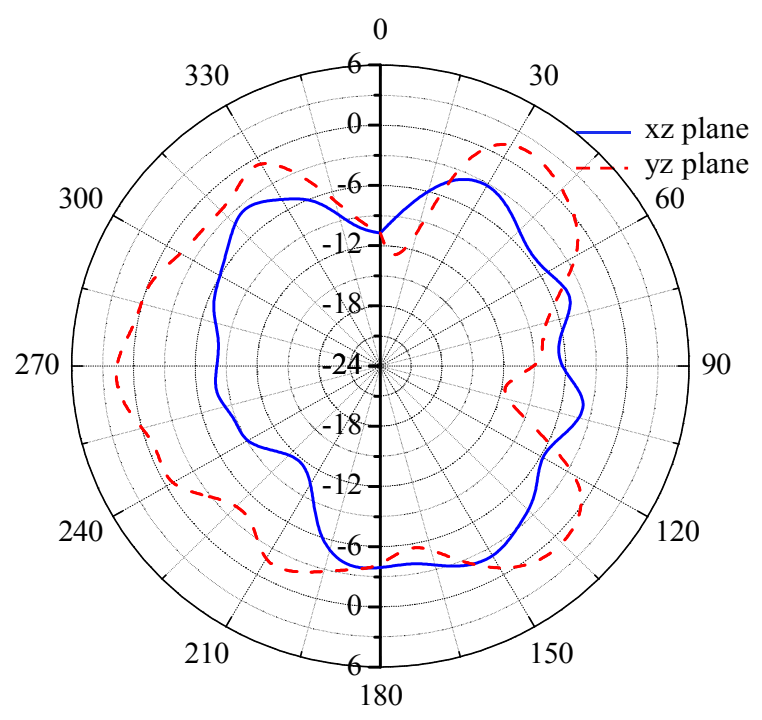

(e)

Figure 7. Radiation patterns of the antenna: (a) at $3.1 \mathrm{GHz}$; (b) at $5.0 \mathrm{GHz}$; (c) at $7.0 \mathrm{GHz}$; (d) at $9.0 \mathrm{GHz}$; (e) at $10.6 \mathrm{GHz}$ 


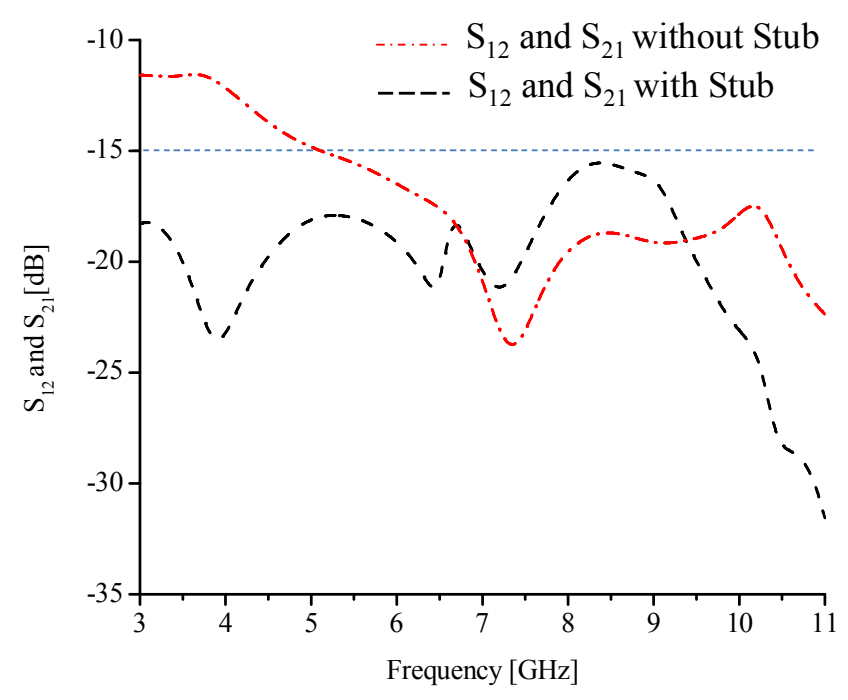

Figure 4. $S_{12}$ and $S_{21}$ of MIMO antennas.

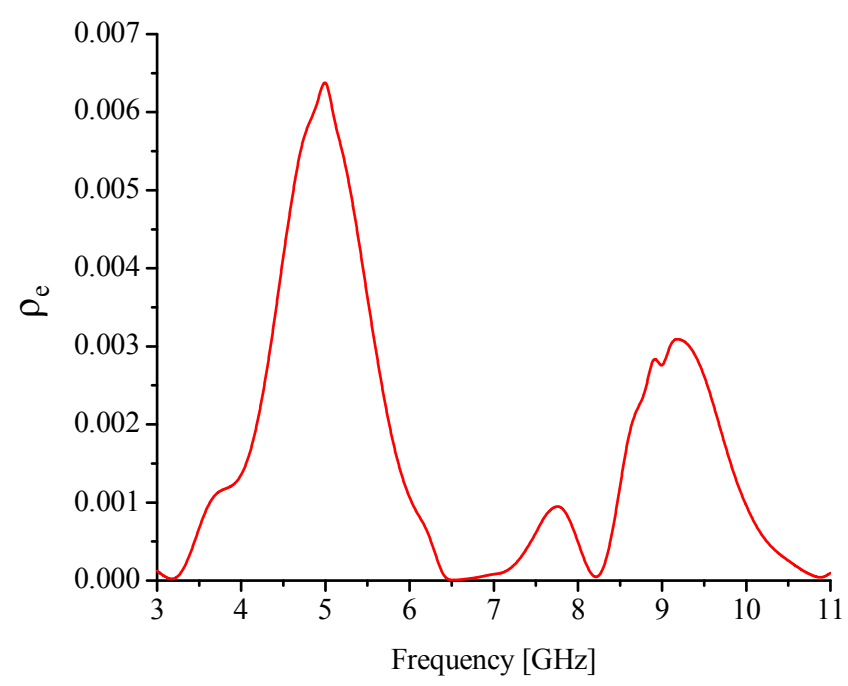

Figure 5. Mutual coupling ratio between two antennas in MIMO.

range; however, it still satisfies the requirements for a MIMO UWB antenna.

Figure 8 depicts the peak gain of the MIMO antenna within the investigated frequency range. It is apparent that although the antenna peak gain varies with frequencies, it is still higher than $3.8 \mathrm{dBi}$ within the frequency range from $3.1 \mathrm{GHz}$ to $10.6 \mathrm{GHz}$. The antenna gain achieves its peak value of $6.8 \mathrm{dBi}$ at the frequency of $11.0 \mathrm{GHz}$.

\section{Measured Results and Discussions}

Based on the simulated results, a prototype has been fabricated as shown in Figure 9.

The VSWR of the two elements were measured and compared with simulation results as shown in Figures 10 and 11. From these figures, it can be seen that the actual and simulated VSWR are less than 2 in the whole investigated frequency range. This shows that the proposed MIMO antenna satisfies the requirements

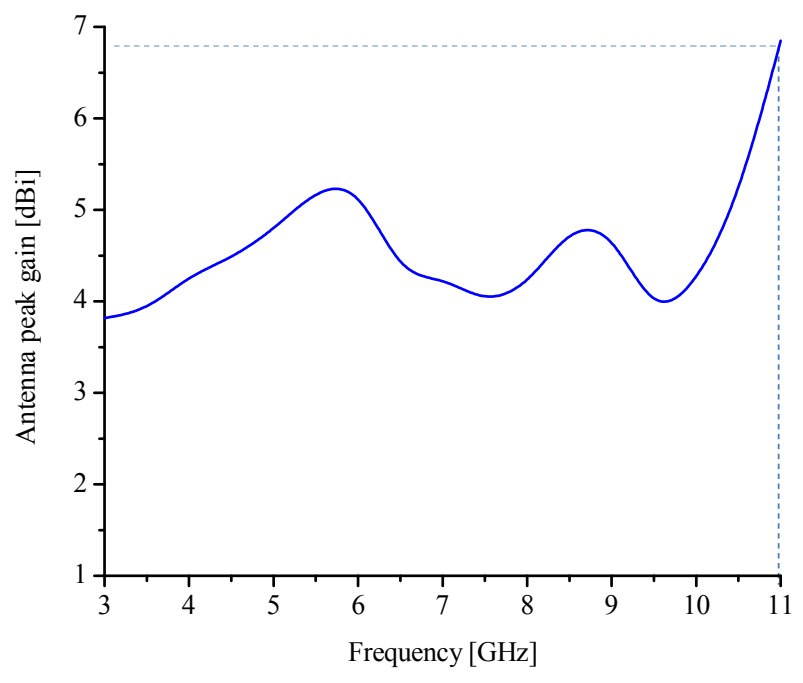

Figure 8. Antenna peak gain versus frequency.

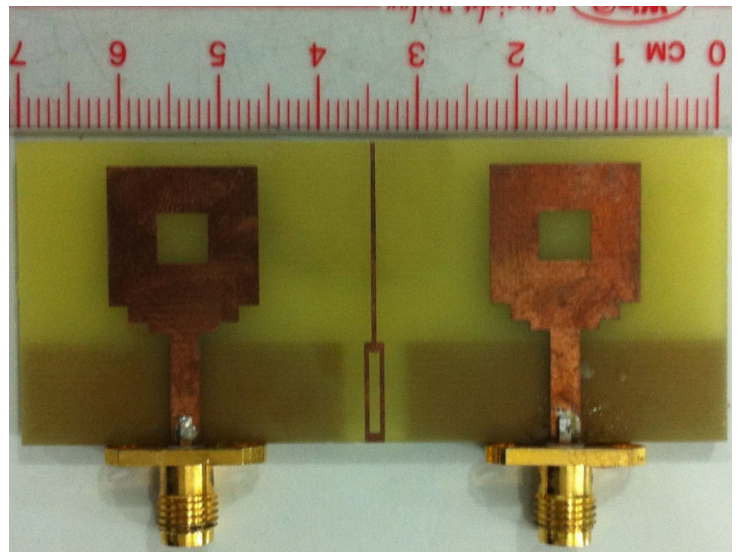

(a)

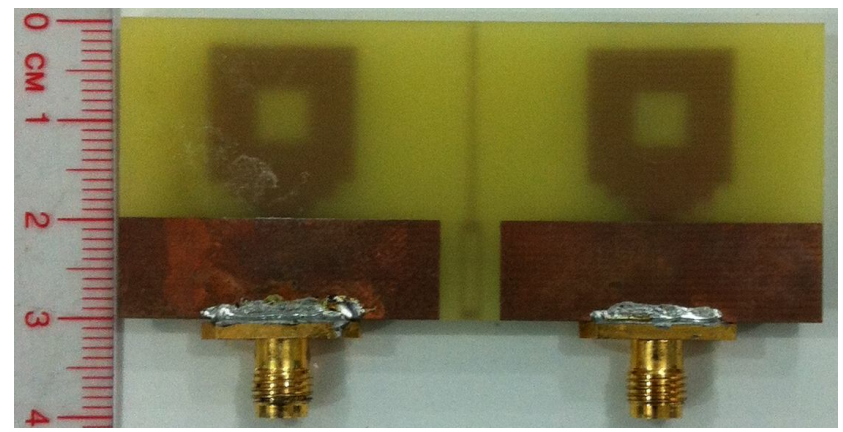

(b)

Figure 9. Photograph of the fabricated MIMO antenna (a) top view; (b) bottom view.

\section{of MIMO UWB antennas.}

In order to evaluate the mutual coupling effect between two antennas, the parameters $S_{12}$ and $S_{21}$ were investigated. Since the two elements in the MIMO antenna have the same structure parameters, $S_{12}$ and $S_{21}$ have the same values. Measurement results and simulation results of $S_{12}$ and $S_{21}$ are shown in Figure 12.

From Figure 12, it can be seen that the measurement result line has the similar shape to that of the simulation 


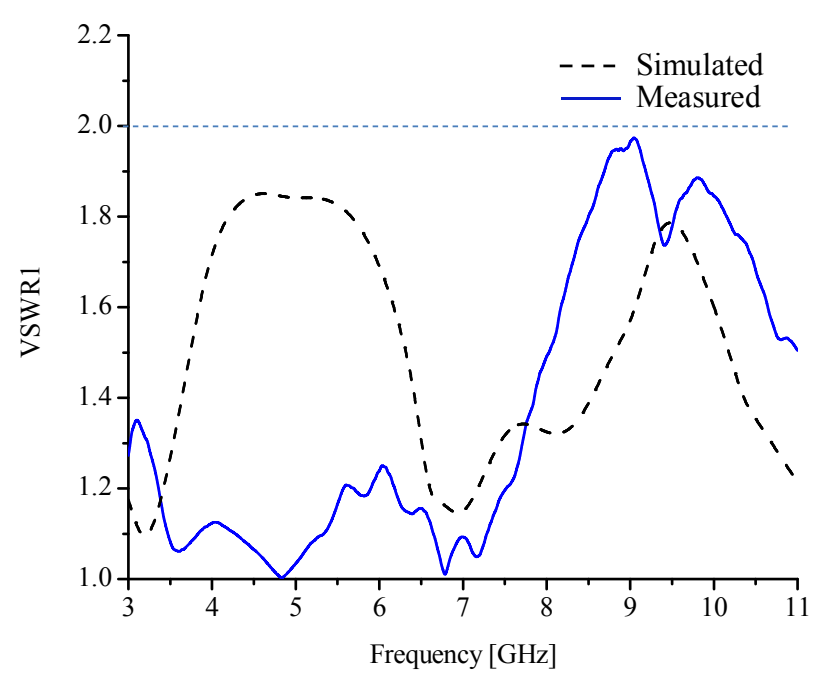

Figure 10. Comparison between measurement results and simulation results of antenna 1 (VSWR).

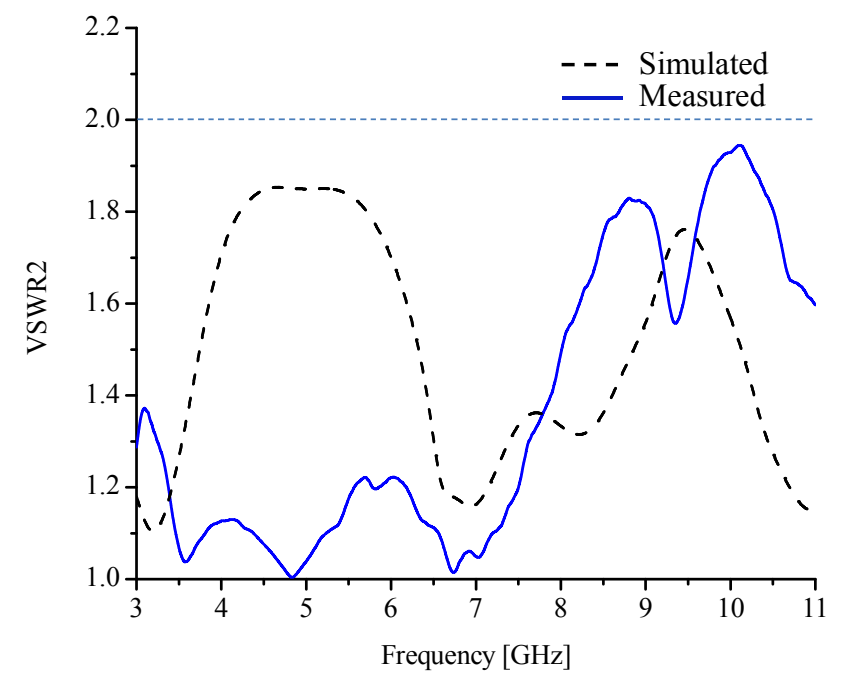

Figure 11. Comparison between measurement results and simulation results of antenna 2 (VSWR).

result. In spite of minor differences, it is still clear that S12 and S21 are less than $-15 \mathrm{~dB}$ in the whole frequency range. This again satisfies the requirements for MIMO UWB antennas to get low mutual coupling effect and work stably within the desired frequency range.

To sum up, the measurement results of the prototype of proposed MIMO UWB antenna agree well with the simulation results. This affirms that all parameters of the proposed antenna satisfy the technical requirements for MIMO UWB antennas with a small size and a low mutual coupling effect.

\section{Conclusion}

This paper has proposed a MIMO UWB antenna with a small size and low mutual coupling, which has some following advantages:

i) The antenna structure is small, compact, simple,

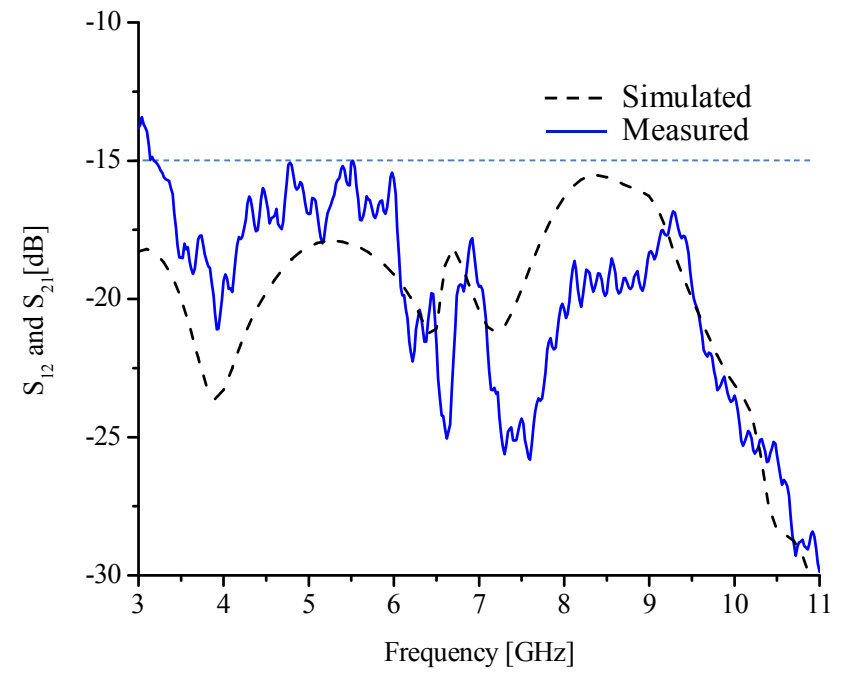

Figure 12. Comparison of measurement results and simulation results of $S_{12}$ of MIMO antennas.

thus it is convenient for fabricating by printed circuit technology.

ii) The VSWR is less than 2 in the whole investigated frequency range from $3.0 \mathrm{GHz}$ to $11.0 \mathrm{GHz}$, which corresponds to $116.8 \%$ of the center frequency.

iii) In spite of ultra-wide frequency range, radiation pattern in the whole frequency range can be considered isotropic.

iv) The peak gain that the antenna achieves in the overall UWB frequency range varies from $3.8 \mathrm{dBi}$ to $6.8 \mathrm{dBi}$.

v) The mutual coupling effect between antennas is relatively small, ensuring its stability.

vi) Measurement results of proposed antenna prototype are similar to simulation results, showing that the proposed antenna meets the requirements for MIMO UWB antennas.

\section{ACKNOWLEDGMENT}

This research was funded by Vietnam National Foundation for Science and Technology Development (NAFOSTED) under grant number 102.01-2012.19.

\section{REFERENCES}

[1] T. A. Rahman, Ed., Reconfigurable ultra wideband antenna and development for wireless communication. Research Project Vot No 79028, Wireless Communication Centre Faculty of Electrical Engineering University Teknology Malaysia, 2008.

[2] M. E. Bialkowsk and A. M.Abbosh, "Design of uwb planar antenna with improved cut-off at the out-of-band frequencies," IEEE Antennas and Wireless Propagation Lettrers, vol. 7, pp. $408-410,2008$.

[3] R. Eshtiaghi, J. Nourinia, and C. Ghobadi, "Electromagnetically coupled band-notched elliptical monopole antenna for uwb applications," IEEE Transactions on Antennas and Propagation, vol. 58, pp. 1397 - 1402, 2010.

[4] Z. A. Zheng, Q. X. Chu, and Z. H. Tu, “Compact bandrejected ultrawideband slot antennas inserting with $\lambda / 2$ 
and $\lambda / 4$ resonators," IEEE Transactions on Antennas and Propagation, vol. 59, pp. 390 - 397, 2010.

[5] D. T. Le and Y. Karasawa, "A novel compact ultrawideband dipole antenna," in IEEE 6th European Conference on Antennas and Propagation (EUCAP), March 2012, pp. $2815-2818$.

[6] X. N. Low, Z. N. Chen, and T. See, "A uwb dipole antenna with enhanced impedance and gain performance," IEEE Transactions on Antennas and Propagation, vol. 57, no. 10 , pp. $2959-2966,2009$.

[7] Z. N. Chen, T. See, and X. Qing, "Small printed ultrawideband antenna with reduced ground plane effect," IEEE Transactions on Antennas and Propagation, vol. 55, no. 2, pp. $383-388,2007$.

[8] L.-X. Li, S.-S. Zhong, and M.-H. Chen, "Compact bandnotched ultra-wideband antenna using defected ground structure," Microwave and Optical Technology Letters, vol. 52, no. 2, pp. 286-289, 2010.

[9] M. Gopikrishna, D. Krishna, C. Aanandan, P. Mohanan, and K. Vasudevan, "Compact linear tapered slot antenna for UWB applications," Electronics Letters, vol. 44, no. 20, pp. $1174-1176,2008$.

[10] L. T. Trung, N. Q. Dinh, and H. D. Thuyen, "A design of compact ultra-wide band antenna," in IEEE International Conference on Advanced Technologies for Communications (ATC), Oct. 2013, pp. $700-703$.

[11] A. I. Najam, Y. Duroc, and S. Tedjini, "Design of mimo antennas for ultra-wideband communications," in MAnisfestation des JEunes Chercheurs en Sciences et Technologies de l'Information et de la Communication (MajecSTIC 2009), 2009 , pp. 1-7.

[12] J.-F. Li, Q.-X. Chu, Z.-H. Li, and X.-X. Xia, "Compact dual band-notched uwb mimo antenna with high isolation," IEEE Transactions on Antennas and Propagation, vol. 61, no. 9, pp. 4759 - 4766, 2013.

[13] V. N. L. Kumar, M. Satyanarayana, B. Pavan, K. V. Kumar, and A. V. Krishna, "Design of printed rectangular monopole antennas for UWB applications," in International Conference on Radar, Communication and Computing (ICRCC), SKP Engineering College, Tiruvannamalai, TN, India., Dec. 2012, pp. 315 - 318.

[14] W. B. C, Ed., Transmission line design handbook. Artech House Boston, Norwood, MA, 1991.

[15] H. Wheeler, "Transmission-line properties of a strip on a dielectric sheet on a plane," IEEE Transactions on Microwave Theory and Techniques, vol. 25, no. 8, pp. $631-$ 647, 1997.

[16] S. I. and P. Vainikainen, "Estimation of signal correlation in antenna arrays," in 12th International Symposium on Antennas (JINA 2002), Nice, France, Nov. 2002, pp. 24852488.

[17] S. Ko and R. Murch, "Compact integrated diversity antenna for wireless communications," IEEE Transactions on Antennas and Propagation, vol. 49, no. 6, pp. 954 - 960, 2001.

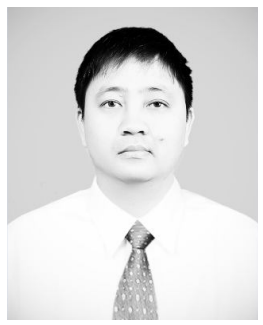

Le Trong Trung received the B.E., the M.E. degrees in Electronic engineering from Le Quy Don Technical University, Ha Noi-City, Viet Nam, in 2006, 2011. He is currently pursuing the Ph.D. degree in Electronic engineering at Department of Fundamental of Radio and Electronic Engineering, Le Quy Don Technical University, Ha Noi-City, Viet Nam.

His research interests include UWB antennas, MIMO antennas.

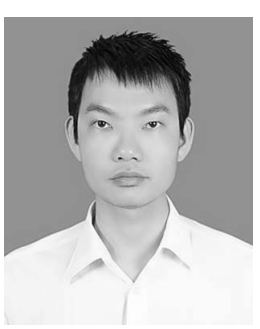

Nguyen Quoc Dinh received the B.E., M.E. and D.E. degrees in Department of Electrical \& Electronic Engineering, National Defense Academy, Yokosuka, Japan, in 2006, 2008, and 2011, respectively. Since 2011, he has been a Research Associate at the Department of Fundamentals of Radio and Electronic Engineering, Le Quy Don Technical University, Hanoi, Vietnam.

His research interests include very small antennas, UWB antennas, MIMO antennas. $\mathrm{He}$ is a member of the Institute of Electronics, Information and Communication Engineers (IEICE), Japan. He was the recipient of the Young Scientist Award of the IEICE Antennas and Propagation Society Japan Chapter, Japan (2011).

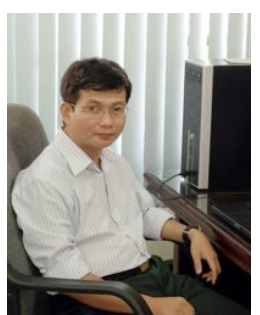

Ta Chi Hieu was born in Vinh Phuc in 1970. He graduated the Military Technical Academy in 1994 with hornor.

He got his MSc degree in electronic engineering in the National Defense Academy of Japan in 2002 and his $\mathrm{PhD}$ degree in signal processing in the University of Strathclyde, United Kingdom in 2008. He is currently working at the Faculty of Radio Electronics, Military Technical Academy.

His research interests include precoding and equalization for MIMO systems, microwave engineering and computational electromagnetics. 